\title{
STUDIES ON THE COLD HEMAGGLUTINATION
}

\author{
KYUHEI NAKADATE* \\ Department of Legal Medicine, School of Medicine, \\ Keio University
}

(Received on Deeember 7, 1955)

\section{INTRODUCTORY}

The cold hemagglutination, or the phenomenon of agglutination brought on the blood cells of men and animals of various kinds at low temperature by sera of their own, of the same blood group, or of other bodies, was first perceived by Hayem (1889) in cases of liver cirrhosis. This was followed by Reitmann (1890) who confirmed Hayem's discovery; by Durham and Blandford (1890) who noted similar phenomena in animals afflicted with trypanosis; by Ascoli and Kanthack in healthy men; and by Landsteiner (1902) in healthy horses, rabbits, cows, guinea pigs and chickens. Since then, such hemagglutination has been studied by many investigators, and proved to occur not only in men and animals in cases of various diseases but also in healthy conditions.

A comprehensive perusal of literature, both in this and other countries, on the cold hemagglutination shows in noteworthy fact that, whereas in foreign countries researches are mostly conducted to find the frequency of its occurence and the agglutination titre in cases of various diseases, in Japan they consist mainly of experimental studies which attempt to ascertain its nature. However, not only the nature of the agglutination, but the process of its fluctuation still remain to be clarified. Recently, Ôba and others carried out studies of the cause of the cold agglutination, and concluded that the cold hemagglutinin is to be considered as a result of autommunization caused by the so-called embryonic antigen contained in the body cells in the early stage of their growth, but their explanation has not yet been generally accepted. There are also many who consider the fluctuation of the cold agglutination to be caused by non-specific stimuli, but they, too, have been unable to supply sufficient proof to win our approval. Consequently, we have been trying to make clear the process of the cold hemagglutination by studying its fluctuations in healthy bodily conditions and in cases of various diseases as well as under varied experimental conditions.

* Professor of Legal Medicine. 


\section{COLD HEMAGGLUTINATION IN HEALTHY MEN AND RABBITS}

1. Frequency of occurence and the titre of the cold autohemagglutination.

Frequency of occurence. The frequency in healthy men has been found to be 47.0-99.7\% by Bond, Amzel, Higuchi, Iwai, Koita, Maeda, Yamashita and others. On the other hand, Yamagami, Kubo, Mizu, Masunaga, Inoue, Kawagoe, Kosugi, and Tsurumi, Saito, Ishibashi, Inatomi, Tanaka, Torigai and others of our laboratory recognized such occurence in all healthy men.

Titre of auto-hemagglutination. Although the maximum titre in healthy men has hitherto been reported to be $16-128 \mathrm{X}$, recent investigations prove that there are some cases of as great as $500 \mathrm{X}$. This is to be attributed to the advanced skill in the method of examination. According to Saito, Torigai and Ishibashi of our laboratory, of 310 healthy men 225 (71.8\%) were 1-50X, $70(23.2 \%)$ were $60-100 \mathrm{X}$, and $6(2.0 \%) 150-500 \mathrm{X}$, titres of $5,10,20$ and $30 \mathrm{X}$ being largest in number. With healthy rabbits, too, the occurence was observed in every case, but the titres vary. Recent report shows, however, that as in healthy men, the titres seem to be mounting. Saito and Morioka of our laboratory place the figures at 5-70X, and most frequently 10-20X.

2. Titre of cold auto-hemagglutination and age.

Higuchi reported that the titre rises with the age, Iwai and Tô that it is not necessarily high in old age, Koita that it is high even in youth, and Maeda that although it is higher in youth than in adult, it again mounts in old age, while Yamashita stated that whereas it rises gradually with the age, it falls when over 60 years old. According to examinations carried out by Saito, Ishibashi and Torigai of our laboratory on 280 healthy men of 15-70 years old, no prominent difference was observed up to 60 , but in old men of over 60 the titre seemed generally to be low.

3. Temperature amplitude of the cold hemagglutination.

Investigators' opinions greatly differ as to the temperature amplitude of the hemagglutination. Tanaka of our laboratory stated that the amplitude lies at $0^{\circ}-20^{\circ} \mathrm{C}$, the occuring in greatest number at $0^{\circ}-15^{\circ}$, followed in order by $0^{\circ}-10^{\circ}, 0^{\circ}-20^{\circ}$ and $0^{\circ}-5^{\circ}$.

With healthy rabbits, the temperature amplitude lies at $0^{\circ}-15^{\circ}$, or somewhat smaller than that of healthy men, and the agglutination is observed in greatest number at $0^{\circ}-15^{\circ}$ and least at $0^{\circ}-5^{\circ}$. The amplitude in healthy men is thus great and the agglutination occurs not only at temperatures as low as $0^{\circ}-5^{\circ}$, as has hitherto been generally believed, but when the temperature is 
$20^{\circ}$. Consequently, it is advisable to pay great attention to the temperature in determining A, B, O blood groups.

4. Velocity of the cold hemagglutination.

According to Tanaka of our laboratory the velocity of the agglutination in healthy men varies with temperature, and the maximum titre is reached in many cases in 5-10 hours at $0^{\circ}-5^{\circ}$, but in some cases the maximum is reached in 2-3 hours. With healthy rabbits, the majority shows the maximum titre in 2-3 hours and in rare cases it requires 10 hours, but on the whole the velocity of the agglutination seems to be greater than that of healthy men.

5. Individual difference of the hemagglutinability.

A perusal of reports hitherto made on the cold hemagglutination shows that in most cases invenstigations were directed to examine only the fluctuation of the cold hemagglutinins or the serum agglutinity, and cases are very rare in which the hemagglutinability was examined at the same time. In investigating the fluctuation of the cold hemagglutination in cases of various diseases, it is difficult to determine whether the titre has risen, remained unchanged or fallen, as the titre in healthy conditions before the affliction is unknown in many cases. The author's experience is, however, that there are cases where the rise or fall is determined with comparative ease by examining the hemagglutinability of the patients by the normal human serum whose titre of the cold auto-hemagglutination is known. For this reason, it is of utmost importanceto determine the individual difference of the hemagglutinability of healthy human. The investigations by Saito and Torigai of our laboratory of the agglutinability brought on the healthy blood cells of all groups (according to the ABO-grouping) by normal human serum the auto-hemagglutination titre of which is known, showed that the difference was at most 2 test-tubes and rarely 4 , by diluting the serum 10 times. Generally speaking, the difference of agglutinability appeares to be greater with the blood cells of group $O$ than that of other groups. The individual difference of the agglutinability of the healthy rabbits blood cells seems to be less pronounced than that of healthy human.

6. Individual difference of the serum agglutinity.

Although accurate determinations of the strengthening and weakening of the cold hemagglutination is difficult, there are cases, as has been alreedy stated, when such determinations are greatly facilitated by examining the hemag. glutinability. Moreover, there are instances when the difficulty is further alleveated by examining, simultaneously with the examination of the hemag- 
glutinability, the serum agglutinity, i.e., the agglutinity of the patients' serum for the blood cells of healthy men whose cold auto-hemagglutination titres are known. According to Saito and Torigai, the difference is usually no more than 1-2 test-tubes by employing $10 \mathrm{X}$ dilution method, and in rare occasions 4 . With healthy rabbits, the results obtained are nearly the same as with healthy men.

7. Seasonal fluctuations.

Ishibashi and Morioka of our laboratory conducted monthly- investigations of the cold auto-hemagglutination titres of many healthy men and rabbits for over one year, and found almost no seasonal fluctuations.

8. Relation between the cold auto-hemagglutination titres and blood groups.

Regarding mutual relations between the cold auto-hemagglutination titres and blood groups A-B-O, Higuchi, Murakami and Maeda concluded that, whereas the agglutination titres are great in sera of group $O$, those of group $A B$ are small. Yamashita claimed that there is no relation between the agglutination titres and the groups of serum. Saito and Torigai made investigations on 150 healthy men, and found no definite relations between the two. Moreover, they found no mutual relations between the agglutination titres and the cold hemagglutination titres.

\section{COLD HEMAGGLUTINATION IN VARIOUS DISEASES}

1. Pulmonary tuberculosis.

Investigations of the cold hemagglutination and the blood sedimentation rate were carried out on pulmonary tuberculosis patients divided into 3 groups of slight, moderate and serious cases, according to $\mathrm{X}$-ray findings, bacillus-detection of the sputum, and other general symptoms. The results showed the averages of the cold auto-hemagglutination titres to be $18.8 \mathrm{X}$ in the group of slight cases (19 patients), $31.3 \mathrm{X}$ in moderate cases (21), and of the group of serious cases (29), 34.3X in survival (19) and $185 \mathrm{X}$ in fatal cases (10). The average medians of the blood sedimentation rates were $19.0 \mathrm{~mm}$ in the slight and $51.0 \mathrm{~mm}$ in the moderate group, while of the serious group the figures were $67.0 \mathrm{~mm}$ in survival $72.0 \mathrm{~mm}$ in fatal cases. These figures seem to prove that with tuberculosis patients the cold hemagglutination titres are generally low in the group of slight cases, high in moderate and serious cases, and especially high in fatal cases. In general, the blood sedimentation rates increase in parallel with the rise of the hemagglutination titres, although the author agrees with Inoue's opinion that this does not always apply to individual cases. Also, when the patients' conditions grow worse, and prognosis unfavorable, the agglutination 
titres rise increasingly, and with their rise a marked increase in the blood sedimentation rates is observed. But in the final stage they show an obvious fall, even, it seems, lower than when in healthy conditions. When the conditions improve and the patients begin to recover, the aggletination titres are known generally to show low figures. Further, investigations of the hemagglutinability of tuberculosis patients (63. cases) showed that there was no case showing a trend to rise, in 5 cases there was a trend of falling, and in the majority no change was observed. But in the determination of the hemagglutinating potency cf the serum (20 cases examined), there were 5 cases which showed a trend to rise, 1 case which seemed to indicate a fall, while in the remainder there seemed to be no change. Consequently, it may be presumed that the rise in the cold hemagglutination in tuberculosis patients in mainly due to the rise in the serum agglutinity, or the increased production of agglutinins, as a result of stimulation on the reticulo-endothelial system. And such stimulation on the reticulo-endothelial system is assumed to be caused by some unknown poisonous substance produced in the bodies of tuberculosis patients.

\section{Syphilis.}

Investigations of the cold hemagglutination in 56 cases of syphilis patients, on whom both the Wassermann and Murata reactions returned strong positive results, showed that in 20 cases $(51.8 \%)$ the auto-hemagglutination titres were $10-50 \mathrm{X}$, in 21 cases $(37.5 \%) 60-100 \mathrm{X}$, and in 6 cases $(10.7 \%$ ) $150-200 \mathrm{X}$, from which it is evident that the titres are high compared with healthy men. Detections of the hemagglutinability of the erythrocytes and the hemagglutinating potency of the serum showed that there were 30 cases (54\%) in which the figures seemed to rise, with an average auto-hemagglutination titre of $90 \mathrm{X}$. In 26 cases (46\%) no changes were observed, and their averate was $30 \mathrm{X}$. These results prove that the cold hemagglutination in syphilis patients does not always show a rise, as hitherto reported by many investigators. Also, it has become definite that parallelism does not exist between the intensity of positivity in the Wassermann or precipitation reactions and the rise in the agglutination. As evidenced by Kita's reports, such rise in the cold hemagglutination in syphilis patients may be attributed to the decline of the antidoting function in the living organisms, especially that of the liver, caused by unknown poisonous substance produced in their bodies, which stimulates the blood formation organs and the reticuloendothelial system.

3. Pleurisy.

Investigations were conducted to determine the cold auto-hemagglutination 
titres and the blood sedimentation rates in 6 eases of moist pleurisy and 1 case of dry pleurisy, and showed the maximum agglutination titre to be $1000 \mathrm{X}$, the minimum $32 \mathrm{X}$, with the average of $310 \mathrm{X}$, the figures markedly higher than in healthy men. The medians of the blood sedimentation rates were $7.7-73.0 \mathrm{~mm}$, and their average $37.98 \mathrm{~mm}$. In the majority of cases, the rise of the cold auto-hemagglutination titres were in parallel with the increase in the sedimentation rates.

\section{Asthma.}

Although the cases examined were few, it was observed that there was generally an intensification in the cold hemagglutination in asthmatic pateints.

\section{Nephritis.}

The cold auto-hemagglutination titres in 7 cases of acute nephritis were investigated, and the results were one case each of $256 \mathrm{X}, 128 \mathrm{X}, 32 \mathrm{X}$, and $16 \mathrm{X}$, and 3 cases $64 \mathrm{X}$, showing no markedly high titres although generally somewhat higher than in healthy men. Inoue reported that nephrosis patients show conspicuously high titres as compared with patients of other renal diseases, and of the 8 cases examined 2 were $2018 \mathrm{X}$, one was $1024 \mathrm{X}, 4$ were $128 \mathrm{X}$, and the remaining one case $32 \mathrm{X}$. Such fact that a rise in the hemagglutination titres in observed in the cases of renal diseases, and especially where the degeneration or inflammatory changes are supposed to be prominent, may be accounted for by the production of some unknown poisonous substance in the bodies of the patients.

6. Cerebral hemorrhage.

7 cases of cerebral hemorrhage were investigated, with the results that among patients of old hemorrhage those accompanied by motor impairments and hypertension generally showed high agglutination titres, and in the cases where the titre showed an inclination to rise, recurrences were observed. When the titres rise still further, the patients proved to be lethal in many cases. On the other hand, if the titres fall gradually after fits, the conditions of the patients begin to improve, and generally tend to recovery. When fresh fits occur, and the titres rise, the prognosis is unfavorable, but if the titres fall, favorable conditions seem to follow.

\section{Tumors.}

16 cases consisting of 4 cases carcinoma of the breast, 2 of the uterus, 2 of the stomach, 1 each of the rectum and skin, 2 sarcoma and 4 utcrine myoma, were examined with the result that the titres of the cold auto-hemagglutination 
were high in many cases of malignant tumors, and that they seem to fall gradually'after the operations. In the cases of uterine myoma which is benignant the titres are also slightly high, and show a gradual fall, similar to cases of malignant tumors, after operations; there being no marked differences between the titres whether the tumors are malignant or benignant. Accordingly, it may be supposed that in both kinds of tumors the governing factor is the degree of the pathological change and cachexy. The intensification of the hemagglutination in the cases of tumors is believed to afford a good background for the hypothesis that the cause of cachexy is an autointoxication.

\section{FLUCTUATION OF THE COLD HEMAGGLUTINATION UNDER VARIOUS CONDITIONS}

Since Kambe reported the production of the cold hemagglutinin in rabbits by injecting raw cow-milk, a large number of investigations have been carried out on the fluctuation of the hemagglutination. Ôba and others recognized the production of the cold hemagglutinin by immunizing with the young bone-marrow of rabbits, blood cells of rabbit's and guinea pig's embryos and lipoids of cells of malignant tumors, and claimed that the cold hemagglutinin is the product of autoimmunization of entire body cells during the early stage of their growth by embryonic lipoid antigens. As to the process of the increase of the agglutinin, there has been no agreement in the views of various investigators; they generally believe the pre-existence of the agglutinin and explain that the intensified production of the agglutinin is due to accidental non-specific stimuli. But many investigations hitherto made were unable to clarify the process of the fluctuation of the hemagglutination. Moreover, the process of the rise in the hemagglutination in the cases of various diseases in men still remains unexplained. It is for this reason that we started to investigate the fluctuation of the hemagglutination under various experimental conditions and at the same time to explain the process of the fluctuation.

1. Liver impairments.

It is evident from Inoue's report that the cold hemagglutination is particularly intensified in the cases of liver cirrhosis compared with other cases. It is, therefore, easily supposed that there exists a close relation between liver impairments and the fluctuation of the hemagglutination. When the liver is impaired with liver poisons: carbon tetrachloride, chloroform or yellow phosphor, the cold hemagglutination fluctuates with the degree of impairment. If a large amount of these liver poisons is injected subcutaneously, the cold autohemagglutination titre, the hemagglutinability of the erythrocytes and the hemagglutinating 
potency of the serum agglutinity fall simultaneously 24 hours after the treatment and most of the animals die. In cases of injecting the moderate dose, the titre rises markedly. and does not return to the normal easily. The sodium santoniate test in which the liver function is examined by the amount of the reagent excreted into urine, shows, in cases of injecting a moderate dose, a marked decrease in the excretion which indicates an obvious impairment of liver function, especially the decline of the antidoting ability. From these results, it is assumed with ease that these is a close relation between the fluctuations of the cold hemagglutination and of the antidoting ability of the liver.

2. Renal impairments.

It has already been stated that a prominent intensification of the cold hemagglutination is observed in the cases of acute nephritis, especially: of nephrosis. By the subcutaneous injection of a large amount of sublimate, which is a kidney poison, a tendency for a slight fall is observed after 24-72 hours; and if a moderate amount is injected, the agglutination titre rises. But both rise and fall are slight in degree compared with the cases in which liver poisons are applied.

3. Injection of shock poisons.

Intravenous injections of 'so-called shock poisons, such as peptone, histamine or acetylcholine, cause a fall in the cold auto-hemagglutination titre, hemagglutinability of the erythrocytes and hemagglutinating potency of the serum agglutinity same as with liver poisons, if a large dose is applied; and cause a rise if the amount applied is moderate. In cases of using acetylcholine, both rise and fall are very slight compared with the cases of peptone and histamine. Therefore, the intensification of the agglutination in the cases of applying moderate amounts may be attributed to the stimulated blood formation organs and reticuloendothelial system owing to the decline of the antidoting ability of the living organism, and the fall in the cases of large amounts to the so-called toxically paralysed state of these organs caused by excessive stimulation.

4. Constriction- and contusion-shock.

Observations taken on rabbits, whose hind legs were bound tightly around the thigh by the Esmarch'e bandage for 3-5 hours and then loosened, show that the cold auto-hemagglutination titre reaches its maximum on the first to second day, then falls gradually to below the normal on 4th to 8th day, and returns approximately to the normal after the ensuing several days. If both hind legs are knocked, the titre rises markedly after 24 hours and in many cases returns approximately to the normal after 72 hours. 
5. Scalds and burns.

When scalds are caused on the ears of rabbits by soaking them in hot water for 3 minutes, the cold auto-hemagglutination titre, the hemagglutinability of the erythrocytes and the hemagglutinating potency of the serum rise gradually from the next day and do not easily return to the normal.' Burns of the third degree of skin or organs (liver, testis), cause a gradual rise from the next day, which do not return to the normal even after two weeks. These results seem to give a favorable backing to the hypothesis that the nature of death from scalding or burns may be an autointoxication.

6. Injection of Cytotoxin.

When a certain amount of extract of rabbit's lung or cerebrum is injected intravenously, the cold auto-hemagglutination titre rises after 24 hours and returns to the normal on 5 th to 7 th day approximately.

7. Injection of the testicular lipoid.

As has been already mentioned, Ôba and others recognized the production of the cold hemagglutinin by immunization with testicular lipoid of rabbits, cows and pigs; and claim that the agglutinin is to be concluded as the product of autoimmunization caused by embryonic lipoid antigens. When the immunization is made on rabbits with testicular lipoid of guinea pigs added with pig serum, the ability of flocculation and complement fixation is obtained and the Forssman's antigen produced, and at the same time a rise of the cold auto-hemagglutination titre is observed. But the rise of the hemagglutination titre is not so prominent as that of the production of lipoid antibodies of these three kinds. Moreover, whereas the lipoid antibodies decrease step by step and day by day, and diminish at last when the injection of antigens is discontinued, the cold auto-hemagglutination titre falls for some time, then returns to the normal, showing a wavy curve. Further, the increase of the cold auto-hemagglutination titre is not in parallel with that of these lipoid antibodies, and in the manner of fluctuation, too, there is a marked difference between the two. When rabbits are injected with testicular lipoid of guinea pigs alone or with $10 \%$ inactivated pig serum, no rise of the hemagglutination titre is observed. With rabbits which have been immunized with the cardiac lipoid of guinea pigs added with pig serum, no rise of the cold auto-hemagglutination titre is observed, although an ability of flocculation and complement fixation, and the Forssman's antigen are produced. But it is to be added that the cold hemagglutinin of rabbit's serum, -immunized with the testicular lipoid of guinea pigs with an addition of pig serum, can not be 
absorbed by the testicular lipoid of guinea pigs. In view of these results, it is clear as claimed by Oba and others that the rise of the cold auto-hemagglutination titre is obviously due to the production of the cold hemagglutinin, but they do not afford sufficient ground for the assumption that such agglutinin is the product of immunization against embryonic antigen which exists in the testicular lipoid of guinea pigs. If the antigen for the cold hemagglutinin is contained in the testicular lipoid as has been stated by Oba and others, a new contrivance will be needed in preparing an antigen which is capable of displaying such antigenity in the living organism. But, as the preparation of such antigen has not yet been actuallized, the right conclusion seems to be that the intensification of the cold hemagglutination is caused by the infliction of non-specific stimulus on the living organism which is caused by the toxity of the compound of guinea pig's testicular lipoid and pig serum.

8. Injection of the hetero-serum, egg white and raw cow-milk.

When rabbits are injected intravenously with inactivated serum of horses, pigs and cows, the cold hemagglutination declines prominently after 24 hours if the amount of serum is large, and most of them die, whereas no change is observed if the amount is small. In the cases of injecting a moderate amount a marked intensification is observed. With injections of certain amount of egg white and raw cow-milk, an intensification is observed as with the hetero-serum of a moderate amount. Moreover, the intensification of the hemagglutination is attributed to the rise of both the hemagglutinability of the erythrocytes and the hemagglutinating potency of the serum agglutinity. It is supposed that, whereas a small dose of foreign proteins, injected intravenously, is completely antidoted in the living organism and causes no change in the cold hemagglutination, a moderate dose is antidoted incompletely and causes an intensification by moderately stimulating the blood formation organs and the reticulo-endothelial system, and a large dose brings about a decline due to the so-called toxic paralysis resulted from an excessive stimulus.

9. Sensitizing with the hetero-serum.

Investigations of the fluctuation of the cold hemagglutination and the liver function in rabbits which were sensitized by transfusing $2.0 \mathrm{cc}$ of horse or cow serum into the ear vein and the abdominal cavity alternatively 3 times each (applying Schittenhelm's method), show a marked rise in the cold hemagglutination; it reaches the maximum at the end of the 2nd week, begins gradually to fall at the end of the 3rd week and at the end of the 5th week returns approximately to the normal which was estimated before the sensitizing. The intensifi- 
cation of the cold hemagglutination results from the rise of both the hemagglutinability of the erythrocytes and the hemagglutinating potency of the serum. In order to estimate the liver functon in sensitized rabbits, the sodium santoninate test was conducted; its excretion into urine showed the maximum decrease about a week after sensitizing. Consequently, the intensification of the cold hemagglutination seems to occur about a week later than the time at which the maximum decline of the liver function is reached.

10. Injection of adrenalin.

It has been assumed by many investigators that a rising tendency of the cold hemagglutination is observed in hypertension patients, and that, experimentally, successive injection of adrenalin, feeding on cholesterin or administration of vitamin $D$ can cause arteriosclerosis. When a certain amount of

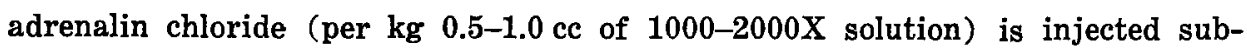
cutaneously for 1-2 weeks in succession, the cold hemagglutination begins to intensify on the 7 th to 10 th day, keeps rising prominently even after 8 weeks following the first injection, and does not return to the normal easily. This fact seems to be resulted from the decline of the function of the liver which is an antidoting organ.

11. Injection of sulfonamide and salvarsan.

Intravenous injections of "Albasil", a sulfonamide, or "Neo-Arsaminol", a salvarsan, cause a fall in the cold hemagglutination if the amount applied is large, and, if moderate, a marked rise. These rise and fall are due respectively to the rise and fall in the hemagglutinability of the erythrocytes and the hemagglutinating serum.

12. Anemia.

1) Anemia caused by bloodlettings: When $10 \mathrm{cc}$ bloodletting is made by venous or cardiac puncture every other day, a slight rising tendency in the cold hemagglutination is observed from about 7th letting. When $20 \mathrm{cc}$ of blood is let every day, the titre once rises on 5th to 9th day, then falls markedly from about 10th day. When the blood is let in an amount of as large as $30 \mathrm{cc}$ per $\mathrm{kg}$ only once, the titre falls prominently from the following day and returns approximately to the pre-treatment normal on 6 th to 14th day.

2) Anemia caused by saponin: When a definite amount of saponin ( $5 \mathrm{mg}$ per $\mathbf{k g}$ ) is injected into the vein, the cold hemagglutination is greatly intensified and reaches its maximum on about 4th day, then falls gradually and returns to the normal on about 20 th day. 
13. Splenectomy.

The cold hemagglutination begins to rise on 2nd to 4th day after splenectomy. and does not return to the normal even on 13th day. The rise in the agglutination is due to the rises of both the hemagglutinability of the erythrocytes and the hemagglutinating potency serum. The control case, in which only the laparotomy is made, shows no rise in the agglutination. These facts may be attributed to the stimulation of the blood formation organs and the reticuloendothelial system by the poisonous substance produced within the body owing to the decline of the antidotic function by splenectomy.

14. X-radiation.

Opinions about the cause of the X-ray sickness still differ. It is as yet unclarified, though theories of gas poisoning, abnormal electrization, abnormal metabolism, endocrine abnormality, disorder of the vegetable nervous system, anaphylaxis, etc. are cited. In order to get a general view of its cause, the cold hemagglutination of rabbits was examined after radiating them with various doses in the liver region. The results were: scarcely any change was seen from the following day to the 10 th day when $150 \gamma$ was radiated; in the case of $300 \gamma$, a prominent rise resulted from the following day and returned approximately to the pre-treatment normal on 7 th to 10 th day; and, when $600 \gamma$ was radiated, three different results were obtained, namely, a prominent fall, a prominent rise followed by a sudden fall to below normal, and a gradual rise. These results are believed to afford an effective background for the theory which attributes the $\mathrm{X}$-ray sickness to intoxication.

15 Ultraviolet radiation.

Rabbits were radiated for 30 minutes in the liver region at a distance of $30 \mathrm{~cm}$ using the Harase's cold quartz lamp. The cold hemagglutination fell prominently immediately after the radiation, then rose gradually, returning approximately to the pre-treatment normal on 3rd to 7 th day.

\section{Starvation.}

In rabbits completely starved, the cold hemagglutination showed no change until 5th day after the commencement of starvation, then fell gradually as the degree of starving advanced, followed by a marked decline in the final stage. The hemagglutinability of the erythrocytes and the hemagglutinating potency of the serum also fell in parallel with each other. Although there is no definite opinion about the nature of the starvation death, the hypothesis that it is caused by autointoxication, and not by a mere lack of nutrients, is gaining 
general acceptance. It is believed that a prominent fall in the cold hemagglutination during starvation has contributed much to this hypothesis.

17. Pregnancy, puerperium and the embryo.

In rabbits, the titre of the cold auto-hemagglutination and the hemagglutinability of the erythrocytes rise prominently in the early stage of pregnancy. They rise more in the middle stage and keep rising even in the final stage. In puerperium, they fall as early as 3rd day and return approximately to the normal of before pregnancy on 7 th day in most cases.

There is no cold hemagglutinin in the serum of rabbit's embryos 22-31 days after conception. It appeares only at the end of 2 months after birth. On the other hand, the hemagglutinability of the erythrocytes of embryos is conspicuously discernible 22-31 days after conception, but it tends to fall as the number of days after conception increases, getting closer to that of the blood cells of grown-up rabbits. The hemagglutinability of the erythrocytes of pregnant rabbits is generally grater than that of embryos. The fact that the cold autohemagglutination titre and the hemagglutinability of the erythrocytes show a marked rise, therefore, may be explained by the stimulation of the blood formation organs and the reticuloendothelial system by the unknown poisonous substance produced within the body during pregnancy, which can not be completely antidoted. Their conspicuous rise during the middle of final stage of pregnancy is probably due to the production in a large quantity of the poisonous substances. Whether the rise in the hemagglutinability of the erythrocytes of the embryos is due to the cause similar to that in pregnant rabbits, namely, the stimulation of the embryonic blood formation organs by poisonous substances of the pregnant rabbits which are transfused through placenta into the embryos; or to the specificity of the embryonic blood cells, as claimed by Oba and others, cannot be as yet fully concluded but it may be attributed to the stimulation of the blood formaion organs of the embryos as in the pregnant rabbits.

\section{RELATIONS BETWEEN THE FLUCTUATION OF THE COLD HEMAGGLUTINATION AND THE BLOOD SEDIMENTATION RATE}

1) No mutual relation is perceived between the cold autohemagglutination titre and the blood sedimentation rate in healthy rabbits.

2) No close mutual relation is observed between the two in healthy men and pulmonary tuberculosis patients. In severe cases of pulmonary tuberculosis, however, there are some instances of their parallelism, as stated before.

3) Intravenous injection of a large quantity of "Albasil", a sulfonamide, brings about a prominent fall in the cold hemagglutination, but causes a great 
increase in the sedimentation rate. There is no great difference between the sedimentation rates at $0^{\circ}$ and $37^{\circ}$. Similar results are obtained when allarge amount of "Neo-Arsaminol" is injected. When a moderate dose of "Albasil" is injected, the cold hemagglutination rises prominently and the sedimentation rate is also greatly accelerated. Moreover, the sedimentation rate at $0^{\circ}$ is slightly higher than at $37^{\circ}$. The results are similar when a moderate dose of "NeoArsaminol" is used. The fact that both the cold hemagglutination and the sedimentation rate rise when a moderate dose of "Albasil" or "Neo-Arsaminol" is injected, and the sedimentation rate is slightly higher at $0^{\circ}$ than at $37^{\circ}$ seems to lead to the recognition of a mutual relation between the two. But, when a large dose is injected the agglutination falls markedly, and the sedimentation rate, on the other hand, is greatly accelerated as in the case of injecting a moderate dose, favoring a denial of the existence of mutual relation.

\section{RELATIONS BETWEEN THE FLUCTUATION OF THE COLD HEMAGGLUTINATION AND THE FUNCTION OF THE RETICULO-ENDOTHELIAL SYSTEM}

It has already been confirmed that the fluctuation of the cold hemagglutination under various conditions are influenced by the increase or decrease of the cold hemagglutinin and by the intensity of the hemagglutinability of the erythrocytes. Consequently, it can easily be supposed that there exist close mutual relations between the reticulo-endothelial system which plays an important role for the production of the agglutinins and various antibodies and the fluctuation of the cold hemagglutination.

1) Single injection of Indian ink: When a definite amount of Indian ink (made by Maruzen Co., Japan) is transfused once intravenously into rabbits, the cold hemagglutination shows a slight tendency for weakening, very much similar to the cases in which a large amount of vitamin $\mathrm{C}$ is injected hypodermically. In both cases, the injections seem to cause an intensified antidotic function of the reticulo-endothelial system.

2) Injection of Indian ink several times: When a definite amount of Maruzen Indian ink is injected once a day for 5 consecutive days, the cold hemagglutination rises markedly 24 hours after the last treatment and does not return to the pretreatment normal even after 3 days. This result very much resembles that of the injection of a definite amount of "Albasil", "Neo-Arsaminol", liver poisons, shock poisons or foreign proteins, or that of burns, pregnancy, $\mathrm{X}$-radiations, etc. Such rise in the cases of injecting Indian ink several times, contrary to the cases of single injection, is supposed to be caused by the decline 
of the antidotic function of the reticulo-endothelial system.

3) Single injection of Indian ink followed by that of "Albasil": When a moderate amount of "Albasil" is injected 48-72 hours after a single transfusion of a definite amount of Maruzen Indian ink, there is scarcely any rise in the cold hemagglutination 24-72 hours after the injection of "Albasil", totally differing from the instances in which a moderate amount of "Albasil" alone is injected into healthy rabbits. Such result very much resembles that of the cases in which treatments are made with vitamin $\mathbf{C}$ or $\mathbf{B}_{1}$, glucose or liver lipoid, preceding the injection of a moderate amount of "Albasil". This seems due to the nearly complete antidoting of "Albasil" injected in a moderate amount, owing to the intensified antidotic function of the reticulo-endothelial system by the single intravenous injection of Indian ink.

4) Injection of Indian ink several times followed by that of "Albasil": When a moderate amount of "Albasil" is injected 8 hours after the injections of Indian ink in a moderate amount, made once a day for 5 days, the rise in the cold hemagglutination is prominent compared with that of the cases in which a moderate amount "Albasil" was used without any preceding treatment. This may be explained as follows: By injecting Indian ink several times the antidoting function of the reticulo-endothelial system declines prominently, contrary to the case of injecting only once, and in even more pronounced degree than the decline caused by injecting healthy rabbits with a moderate amount of "Albasil", giving rise to the intensification of the agglutination.

From these results it is obvious that there exist colse relations between the fluctuation of the cold hemagglutination and the function of the reticuloendothelial system. When the function of the reticulo-endothelial cells, in cases of intoxications by "Albasil", salvarsan or carbon tetrachloride, is examined by the Adler--Reimann's Congo red color-index, which is widely applied for examining the function of the reticulo-endothelial cells, an increase of the index is observed; and this shows an obvious decline of the function of the reticuloendothelial cells according to the general perception of the method applied. Moreover, patho-histologically, a marked degeneration of the reticulo-endothelial cells is observed. It seems conflicting greatly with the general opinion about the recitulo-endothelial system that, when "Albasil", salvarsan or carbon tetrachloride are used, whereas a prominent increase of Congo red color-index, i.e., the decline of the function, and the degeneration of the reticulo-endothelial cells are observed, the production of the cold hemagglutinin is actually levated. On the other hand, Inose reported recently that the production of the agglutinin against typhoid bacilli in rabbits whose reticulo-endothelial cells were highly degenerated with 
poisons such as arsenious acid or Janus green did not much differ from that in healthy rabbits. Consequently, the increase of the Adler-Reimann's Congo red color-index is believed to indicate merely the decline of the phagocytic function of the reticulo-endothelial cells. It is further considered that the antibody production of the reticulo-endothelial cells is approximately in parallel with the degree of their degeneration and the increase of the Congo red color-index within a definite extent.

VI. RELATIONS BETWEEN THE FLUCTUATION OF THE COLD HEMAGGLUTINATION AND THE HEMOGRAM

By the injection of "Albasil" in a large amount, the erythrocytes, leukocytes and reticulocytes increase markedly in the early stage, and the erythrocytes decrease in the following stage. By injecting a moderate amount, the change is practically the same as that caused by a large amount, by the increase of the erythrocytes is generally very prominent and does not return to the normal easily. It is believed that such increase of the erythrocytes, leukocytes and reticulocytes in the early stage in cases of injecting a large amount suggests a strong stimulation of the blood formation organs, and that the decrease in the following stage is caused by the decline of their capability of forming new erythrocytes due to their paralysed state by the excessive stimulation in the early stage. It is moreover clear from the changes of the hemograms that the blood formation organs are strongly stimulated by injecting "Albasil" even in a moderate amount. That the increase of the erythrocytes in the cases of injecting a moderate amount is very high compared with that in the cases of a large amount and dces not decline, and that the increase of the reticulocytes in the former cases is slighter in degree than that in the latter cases, seem to be due to the different degrees of stimulation of the blood formation organs brought on by the difference in the amount injected. A comparative survey of the change in the hemogram and the fluctuation of the cold hemagglutination in cases of "Albasil" injection leads to an assumption of the existence of some relation between the increase of the erythrocytes and the rise in the cold hemagglutination, but it is still undecided whether or not the fluctuations of the two are invariably parallel.

VII. RELATION BETWEEN THE FLUCTUATION OF THE COLD HEMAGGLUTINATION AND THE ACIDOSIS OR ALKALOSIS

It is apparent that there is a close relation between the fluctuation of the cold hemagglutination and the acidosis from the fact that injections of "Albasil" in large amount, starvation or bloodlettings in large quantity etc. cause acidosis, 
and, at the same time, a marked depression of the cold hemagglutination. When various quantities of hydrochloric acid are injected and the fluctuations of the cold hemagglutination, the blood plasma $\mathrm{pH}$, the accommodation capacity of the blood plasma $\mathrm{CO}_{2}$ in each case are examined and their mutual relations investigated, the intravenous injections of N/50 hydrochloric acid 2.0-4.00 cc per $\mathrm{kg}$ cause a rise in the cold hemagglutination without any change in the blood plasma $\mathrm{pH}$ and the accommodation capacity of the blood plasma $\mathrm{CO}_{2}$. When $4.0 \mathrm{cc}$ per $\mathrm{kg}$ is injected, the agglutination rises in half of the cases and falls in the other half. When $5 \mathrm{cc}$ per $\mathrm{kg}$ of $\mathrm{N} / 10$ hydrochloric acid is injected, the cold hemagglutination falls in the all cases, but there is no change in the plasma $\mathrm{pH}$ and the accommodation capacity of the plasma $\mathrm{CO}_{2}$. When 5 cc per $\mathrm{kg}$ of $\mathrm{N} / 10$ hydrochloric acid is injected every day into the abdominal cavity, both the plasma $\mathrm{pH}$ and the accommodation capacity of the plasma $\mathrm{CO}_{2}$ decrease markedly from about 3rd day, causing a prominent acidosis, and at this stage the cold hemagglutination shows a conspicuous depression followed in many cases by death: Intravenous injection of $5 \mathrm{cc}$ per $\mathrm{kg}$ of $\mathrm{N} / 50$ hydrochloric acid causes a marked fall in the cold hemagglutination at the end of 24 hours. When this is followed by an injection of a moderate dose salvarsan (quantity which causes a prominent rise in the cold hemagglutination), the agglutination rises but slightly, showing a great difference to the cases when a moderate dose salvarsan alone is injected into healthy rabbits. In cases of alkalosis caused by injecting sodium bicarbonate in various quantities almost no change is observed in the cold hemagglutination.

\section{RELATION BETWEEN THE FLUCTUATION OF THE COLD HEMAGGLUTINATION AND THE LIVER FUNCTION}

It is a well-known fact that there exist impairments of the liver function in cases of liver cirrhosis, syphilis, pulmonary tuberculosis, acute nephritis, allergic diseases, malignant tumors, etc.; and it has already been mentioned that a prominent change in the cold hemagglutination is observed in these cases. Moreover, it has already been ascertained that there exists, in cases of experimental liver impairments, sensitizing with hetero-serum or poisoning of sulfonamide and salvarsan, a decline of the liver function, especially that of the antidoting capability, as proved by the results of charging tests of sodium santoninate or glucose, by the elevated permeability of the coloring matter, the abnormality of the inorganic matter metabolism, etc. From these facts, it is easily discernible that there is an inseparable relation between the cold hemagglutination and the liver function. The above hypothesis is further supported by the fact that the intensification of the cold hemagglutination, especially that 
of the hemagglutinability of the erythrocytes and the hemagglutinating potency of the serum, 'under various conditions to be described later, can be checked almost completely, and the impairment of the antidoting capability of the liver can be hindered to a very marked degree, by using substances which improve the function of the living organism and especially the antidoting capability of the liver, e.g., vitamin $C, B_{1}, B_{2}$, glucose, organ Jipoids, etc.

\section{RISE IN THE COLD HEMAGGLUTINATION AND THE INFLUENCES THEREON OF VITAMIN $C, B_{1}, B_{2}$, GLUCOSE, ORGAN LIPOIDS}

It has been ascertained by the author's co-investigators that the cold hemagglutination rises markedly by the injections of liver poisons (carbon tetrachloride, chloroform, yellow phosphor), shock poisons (peptone, histamine, acetylcholine), organ poisons, hetero-sera, testicular lipoids, egg white, raw cow-milk, adrenalin, sulfonamides, salvarsans, etc., and in cases of constrictionand contusion-shock, scalds and burns, sensitizing with hetero-sera, splenectomy, anemia caused by bloodletting and saponin, $\mathrm{X}$-radiation, pregnancy, etc. They have also ascertained that such rise in the cold hemagglutination can be checked almost completely by vitamin $C, B_{1}, B_{2}$, glucose, organ lipoids, etc. which serve to improve the antidoting capability of the living organism, especially the antidoting ability of the liver and the reticulo-endothelial system. It has further been observed that the general impairments of the liver function and the reticulo-endothelial cells, too, are greatly hindered by the use of these medicines. In considering these facts, it may be presumed that there are close relations between the fluctuation of the cold hemagglutination and the function of the living organism especially that of the liver and the reticulo-endothelial system.

\section{CONCLUSION}

From the foregoing investigations of the fluctuation of the cold hemagglutination, and also that of the hemagglutinability and the serumagglutinity under various conditions, it is presumable that when substances which are considered poisonous, either qualitatively or quantitatively, are produced in or transfused into the body, and are not completely antidoted, they stimulate the blood formation organs and the reticulo-endothelial system, thereby causing a rise in the cold hemagglutination; and when these organs are paralysed by excessive stimuli, a fall results. 